\title{
Standards and Guidelines for CFTR Mutation Testing
}

Carolyn Sue Richards, $\mathrm{PhD}$, Chair ${ }^{1-3}$, Linda A. Bradley, $P h D^{1-3}$, Jean Amos, PhD ${ }^{2}$, Bernice Allitto, $P h D^{2}$, Wayne W. Grody, MD, PhD ${ }^{1,3}$, Anne Maddalena, PhD ${ }^{1,3}$, Matthew J. McGinnis, PhD ${ }^{1,3}$, Thomas W. Prior, PhD ${ }^{1,3}$, Bradley W. Popovich, $P h D^{1,3}$, and Michael S. Watson, $P h D^{3}$

\begin{abstract}
One mission of the ACMG Laboratory Quality Assurance (QA) Committee is to develop standards and guidelines for clinical genetics laboratories, including cytogenetics, biochemical, and molecular genetics specialties. This document was developed under the auspices of the Molecular Subcommittee of the Laboratory QA Committee by the Cystic Fibrosis (CF) Working Group. It was placed on the "fast track" to address the preanalytical, analytical, and postanalytical quality assurance practices of laboratories currently providing testing for CF. Due to the anticipated impact of the ACMG recommendation statement endorsing carrier testing of reproductive couples, it was viewed that CF testing would increase in volume and that the number of laboratories offering CF testing would also likely increase. Therefore, this document was drafted with the premise of providing useful information gained by experienced laboratory directors who have provided such testing for many years. In many instances, "tips" are given. However, these guidelines are not to be interpreted as restrictive or the only approach but to provide a helpful guide. Certainly, appropriately trained and credentialed laboratory directors have flexibility to utilize various testing platforms and design testing strategies with considerable latitude. We felt that it was essential to include technique-specific guidelines of several current technologies commonly used in laboratories providing CF testing, since three of the four technologies discussed are available commercially and are widely utilized. We take the view that these technologies will change, and thus this document will change with future review. Genet Med 2002:4(5):379-391.
\end{abstract}

Key Words: cystic fibrosis, genetic testing, medical genetics, CFTR, molecular diagnostics

\section{CF 1 INTRODUCTION}

Disease-specific statements are intended to augment the current general ACMG Standards and Guidelines for Clinical Genetics Laboratories (http://www.acmg.net). This document is intended to enhance the ACMG statement on Laboratory Standards and Guidelines for Population-Based Cystic Fibrosis Carrier Screening ${ }^{1}$ by inclusion of more technical laboratory issues related to CF testing and including diagnostic and prenatal testing as well as carrier screening. It is intended for genetic testing professionals who are already familiar with the disease and the methods of analysis. Issues related to the trypsinogen component of newborn screening are not addressed, but these guidelines are applicable to subsequent molecular analysis of newborns.

Individual laboratories are responsible for meeting the CLIA/CAP quality assurance standards with respect to appro-

\footnotetext{
${ }^{1}$ Molecular Subcommittee of the Laboratory Quality Assurance Committee, ${ }^{2}$ Cystic Fibrosis Molecular Working Group, and ${ }^{3}$ Laboratory Quality Assurance Committee.

This is the second of a series of disease-specific supplements to the Standards and Guidelines for Clinical Genetics Laboratories of the American College of Medical Genetics.

American College of Medical Genetics, 9650 Rockville Pike, Bethesda, MD 20914-3998.

Go to www.geneticsinmedicine.org for a printable copy of this document.

See disclaimer at the end of this article.
}

DOI: 10.1097/01.GIM.0000031065.72493.BC priate sample documentation, assay validation, general proficiency, and quality control measures.

\section{CF 2 BACKGROUND ON CYSTIC FIBROSIS}

CF 2.1 Gene symbol/chromosome locus: CFTR on chromosome $7 \mathrm{q} 31.2$ was positionally cloned in $1989 .^{2-4}$ CFTR contains 27 coding exons; genomic sequence is $\sim 230 \mathrm{~kb}$; mRNA is $\sim 6.5 \mathrm{~kb}$.

CF 2.2 OMIM number: 602421.

CF 2.3 Brief clinical description: $\mathrm{CF}$ is the most common autosomal recessive disease in the Caucasian population, with a prevalence estimate of 1 in 2500 to 3300 live births. CF is characterized by viscous mucus in the lungs with involvement of digestive and reproductive systems as well as sweat glands (excess salt loss). Pulmonary disease is the critical factor in prognosis/survival, but both pancreatic sufficient and insufficient forms exist. Recurrent and persistent pulmonary infections are common and lead to respiratory failure. Pancreatic insufficiency occurs in $85 \%$ of affected individuals. Neonatal meconium ileus occurs in $10 \%$ to $20 \%$ of newborns with CF. Other manifestations include chronic sinusitis, nasal polyps, liver disease, pancreatitis, and congenital bilateral absence of the vas deferens (CBAVD). The overall average survival of patients with CF, including those with milder presentation, is approximately 30 years. Treatment for patients with CF is palliative and includes control of infections, clearance of mucus in 
the lung, and improvement of nutrition through pancreatic enzymatic replacement. Somatic gene therapy is a research focus. Nonclassic or atypical forms of CF, with later onset or milder presentation, have been described and will be discussed in a later section. For more information see the online GeneClinics profile at www.geneclinics.org and the National Cystic Fibrosis Foundation at www.cff.org.

Newborn screening programs for CF measure immunoreactive trypsin. Differential diagnosis is by sweat chloride testing $(>60 \mathrm{mmol} / \mathrm{L})$.

\section{CF 2.4 Mode of inheritance: Autosomal recessive.}

CF 2.5 Gene description/normal gene product: CFTR, the CF transmembrane conductance regulator, is 1480 amino acids with a mass of $\sim 170,000 \mathrm{D}$. CFTR is in the ATP-binding cassette family of transporter proteins. The CFTR protein contains five domains, including two membrane-spanning domains, a regulatory domain, and two nucleotide-binding domains that interact with ATP.

CF 2.6 Mutational mechanism/abnormal gene product: An abnormal CFTR protein results in defective electrolyte transport and defective chloride ion transport in the apical membrane epithelial cells of the sweat gland, airway, pancreas, and intestine. There are four classes of CFTR mutations: Class I lead to defective protein products; Class II result in defective protein processing; Class III have a defect in the channel regulation; and Class IV are defective in conductance through the channel and represent milder mutations. Mutations in CFTR can affect the function of the cAMP-regulated chloride channel membrane-spanning domains of the CFTR that form the channel pore, or the channel opening, which is controlled by phosphorylation of the regulatory domain residues.

CF 2.7 Mutation spectrum: A complete list of all mutations can be found in the CF Mutation Database at http:// www.genet.sickkids.on.ca. Over 900 mutations have been identified in the CFTR gene; however, the vast majority of mutations are at frequencies of $<0.1 \%$ or represent private mutations. The major mutation, $\Delta \mathrm{F} 508$, accounts for $30 \%-$ $88 \%$ of CF chromosomes worldwide, depending upon race/ethnicity.

CF 2.8 Ethnic association of common mutations: The ACMG recommended carrier screening panel, while panethnic, is primarily based on mutation frequency in the Ashkenazi Jewish and other non-Hispanic Caucasian population due to the high frequency of the disease and the availability of existing data. Laboratories providing testing for black, Hispanic, or other ethnic groups should be aware of mutation frequencies as applies to their testing population. Depending upon the ethnic group, these mutation frequencies may be difficult to obtain (Table 1)..$^{5-7}$

CF 2.8.1 The most common mutations in the Ashkenazi Jewish population have been described. ${ }^{8-10}$ These include W1282X, $\Delta$ F508, G542X, N1303K, and $3849+10 \mathrm{kbC}>\mathrm{T}$. A recent report by Orgad et al. ${ }^{11}$ indicated that additional mutations were found in Jewish Israeli populations, including D1152H, $405+1 G>A$, W1089X, and S549R.
CF 2.8.2 Mutations specific for the African-American population have been described ${ }^{12}$ and include $3120+1 \mathrm{G}>\mathrm{A}$, A559T, G330X, 2307insA, $\Delta$ F311, and G480C.

CF 2.8.3 In general, the term Hispanic refers to persons from Latin America, a wide geographic area of significant racial and ethnic diversity. Latin American countries include the Caribbean (e.g., Jamaica, Puerto Rico, Cuba), Central America (e.g., Mexico, Costa Rica), and South America. Populations from these areas include descendants of Europeans, native people, and Africans/blacks, with variable levels of admixture. For many of these geographical areas, data on mutation frequencies are unavailable; others are based on small studies or limited testing panels. Mutations not included in the 25 mutation core testing panel that have been reported in these populations include D1270N, W1089X, and S549N. ${ }^{13-15}$

CF 2.8.4 Insufficient information is available for the Asian American population.

\section{CF 2.9 Indications for testing}

- Diagnostic testing, possible diagnosis of $\mathrm{CF}$

- Diagnostic testing, definite diagnosis of CF

- Diagnostic testing, infants with meconium ileus

- Diagnostic testing, CBAVD in males

- Carrier testing, partners of individuals with positive family history

- Carrier testing, partners of CBAVD males

- Carrier testing, general population of reproductive couples

- Carrier testing, positive family history

- Carrier testing, gamete donors

- Preimplantation testing

- Prenatal diagnostic testing, positive family history or for couples having a CF mutation in both partners

- Prenatal diagnostic testing, echogenic bowel in fetus during second trimester

- Newborn screening

CF 2.10 Genotype-phenotype considerations: Genotypephenotype correlations are imprecise and should not be used clinically in predicting lung involvement or survival. Mutations in CFTR have been classified on the basis of association with pancreatic sufficient or insufficient phenotype, with nonclassic or atypical CF presentation, including borderline to normal sweat chloride levels, pancreatic sufficiency, male infertility, or mild pulmonary disease. Examples of such mutations include R117H, $3849+10 \mathrm{kbC}>\mathrm{T}$, A455E, $2789+5 \mathrm{G}>\mathrm{A}, \mathrm{G} 85 \mathrm{E}$, and R334W. However, no significant correlation with genotype, or concordance within sibships, has been demonstrated for pulmonary disease. While there is variability in pulmonary phenotype, the majority of individuals with CF have serious, progressive lung disease. Approximately $85 \%$ of patients with CF are pancreatic insufficient. The remainder of patients with $\mathrm{CF}$ 
Table 1

Residual risk for couples without a positive family history of CF to have a child affected with CF

\begin{tabular}{|c|c|c|c|c|c|}
\hline \multirow[b]{2}{*}{ Ethnic group } & \multicolumn{3}{|c|}{ Test result for couple } & \multirow[b]{2}{*}{$\begin{array}{l}\text { Couple's carrier risk } \\
\text { (product of individual risk) }\end{array}$} & \multirow[b]{2}{*}{$\begin{array}{l}\text { Risk for CF child } \\
(\text { couple's risk } \times 1 / 4)\end{array}$} \\
\hline & $\begin{array}{l}\text { Not tested } \\
\text { (carrier freq.) }\end{array}$ & $\begin{array}{l}\text { Negative } \\
\text { (see report) }\end{array}$ & $\begin{array}{l}\text { Positive } \\
\text { (1) }\end{array}$ & & \\
\hline \multirow[t]{2}{*}{ Ashkenazi Jewish } & $\mathrm{XX}^{a}(1 / 25)$ & & & 1 in 625 & 1 in 2,500 \\
\hline & & $\mathrm{XX}(1 / 800)$ & & 1 in 640,000 & 1 in $2,560,000$ \\
\hline \multirow[t]{4}{*}{$(97 \%)$} & & & $\mathrm{XX}$ & 1 & 1 in 4 \\
\hline & $\mathrm{X}$ & $\mathrm{X}$ & & 1 in 20,000 & 1 in 80,000 \\
\hline & $\mathrm{X}$ & & $\mathrm{X}$ & 1 in 25 & 1 in 100 \\
\hline & & $\mathrm{X}$ & $\mathrm{X}$ & 1 in 800 & 1 in 3,200 \\
\hline \multirow[t]{2}{*}{ Non-Hispanic Caucasian ${ }^{b}$} & $\mathrm{XX}(1 / 25)$ & & & 1 in 625 & 1 in 2,500 \\
\hline & & $\mathrm{XX}(1 / 240)$ & & 1 in 58,000 & 1 in 230,000 \\
\hline \multirow[t]{4}{*}{$(90 \%)$} & & & $\mathrm{XX}$ & 1 & 1 in 4 \\
\hline & $\mathrm{X}$ & $\mathrm{X}$ & & 1 in 6,000 & 1 in 24,000 \\
\hline & $\mathrm{X}$ & & $\mathrm{X}$ & 1 in 25 & 1 in 100 \\
\hline & & $\mathrm{X}$ & $\mathrm{X}$ & 1 in 240 & 1 in 960 \\
\hline \multirow[t]{3}{*}{ African American } & $\mathrm{XX}(1 / 65)$ & & & 1 in 4,200 & 1 in 17,000 \\
\hline & & $\mathrm{XX}(1 / 207)$ & & 1 in 43,000 & 1 in 170,000 \\
\hline & & & $\mathrm{XX}$ & 1 & 1 in 4 \\
\hline \multirow[t]{3}{*}{$(69 \%)$} & $\mathrm{X}$ & $\mathrm{X}$ & & 1 in 13,500 & 1 in 54,000 \\
\hline & $\mathrm{X}$ & & $\mathrm{X}$ & 1 in 65 & 1 in 260 \\
\hline & & $\mathrm{X}$ & $\mathrm{X}$ & 1 in 207 & 1 in 830 \\
\hline \multirow[t]{2}{*}{ Hispanic American } & $\mathrm{XX}(1 / 46)$ & & & 1 in 2,100 & 1 in 8,500 \\
\hline & & $\mathrm{XX}(1 / 105)$ & & 1 in 11,000 & 1 in 44,000 \\
\hline \multirow[t]{4}{*}{$(57 \%)$} & & & $\mathrm{XX}$ & 1 & 1 in 4 \\
\hline & $\mathrm{X}$ & $\mathrm{X}$ & & 1 in 4,800 & 1 in 19,000 \\
\hline & $\mathrm{X}$ & & $\mathrm{X}$ & 1 in 46 & 1 in 184 \\
\hline & & $\mathrm{X}$ & $\mathrm{X}$ & 1 in 105 & 1 in 420 \\
\hline \multirow[t]{2}{*}{ Asian American ${ }^{c}$} & XX (1/90) & & & 1 in 8,100 & 1 in 32,400 \\
\hline & & $\begin{array}{c}\mathrm{XX}(<1 / \\
90)\end{array}$ & & $<1$ in 8,100 & $<1$ in 32,400 \\
\hline \multirow[t]{4}{*}{ (?) } & & & $\mathrm{XX}$ & 1 & 1 in 4 \\
\hline & $\mathrm{X}$ & $\mathrm{X}$ & & $<1$ in 8,100 & $<1$ in 32,400 \\
\hline & $\mathrm{X}$ & & $\mathrm{X}$ & 1 in 90 & 1 in 360 \\
\hline & & $\mathrm{X}$ & $\mathrm{X}$ & $<1$ in 90 & $<1$ in 360 \\
\hline
\end{tabular}

The purpose of this table is to present residual risk calculations using Bayesian analysis based on our current data of the carrier frequency of each racial/ethnic group and the sensitivity of the recommended panel of mutations in each respective group. These data were derived from Grody et al. ${ }^{1}$ and based on published data from the CF Genetic Analysis Consortium, ${ }^{5}$ the CF Foundation registry, ${ }^{6}$ and the NIH Consensus reports of $1997 .{ }^{7}$ It is important to recognize that there are limitations to these data and that as new information becomes available, these numbers will be subject to change.

${ }^{a} \mathrm{X}$ indicates test result for each partner of the couple.

${ }^{b}$ The detection rate of $90 \%$ for non-Hispanic Caucasians was determined based on reanalysis of the data from the CF Genetic Analysis Consortium and the CF Foundation. ${ }^{16}$ A CF carrier rate of 1 in 25 for the non-Hispanic Caucasian population (including the Ashkenazi Jewish population) is based upon a prevalence estimate of 1 in $2500 .{ }^{16}$ Note that these numbers are different from those presented by Grody et al., ${ }^{1}$ reflecting reanalysis of existing data and new data.

${ }^{c}$ There is insufficient data for the Asian American population to allow accurate prediction of residual risk following a negative test result. For couples of mixed ethnicity or for individuals having a positive family history, specific calculations will be required. The above table was calculated as follows: (carrier risk of mother) $\times$ (carrier risk of father) $\times(1 / 4)=$ risk of having a child with CF. The carrier frequency in each specific ethnic population is shown under "not tested"; the CF standard test panel sensitivity for each ethnic population is shown under that population in parentheses. 
are pancreatic sufficient and usually have at least one mutation associated with a milder phenotype. It is important to note that there are a number of exceptions to these generalizations.

\section{CF 2.11 Special testing considerations}

\section{CF 2.11.1 Clinical validation: Clinical sensitivity and specificity}

CF 2.11.2 The clinical sensitivity of CFTR testing varies depending on several factors, including the mutation panel being used, the ethnic population being tested, and the clinical setting. Therefore, it is important that the laboratories request information about race/ethnicity, family history, and reason for testing. The following sections provide estimates of clinical sensitivity and specificity for non-Hispanic Caucasians (hereafter referred to as Caucasians including individuals of American Caucasian, Caucasian with mixed European ancestry, and Caucasian with Northern European ancestry), using the ACMG recommended mutation panel for carrier screening in a prenatal and preconceptual setting.

Clinical sensitivity: Clinical sensitivity is defined as the proportion of individuals who have $\mathrm{CF}$ and also have a positive CFTR test with two identifiable mutations. This can be done within the laboratory or through the literature. For example, the proposed panel of 25 mutations will identify about $90 \%$ of mutations in Caucasians. ${ }^{16}$ Thus about $81 \%$ $(90 \% \times 90 \%)$ of Caucasians with CF (or the same proportion of carrier couples) will have a positive test result (two mutations identified). Laboratories should also be able to provide estimated clinical sensitivities for other defined racial/ethnic groups that may be tested. Estimates of clinical sensitivity should also take into account published estimates of analytic sensitivity.

Clinical specificity: Clinical specificity can be defined as the proportion of negative test results among individuals who do not have CF. Analytic error or variable expressivity of certain mutations can reduce the clinical specificity of the test. Although the clinical expression of most of the 25 recommended mutations is known to be highly consistent with a CF phenotype, there may be some exceptions. For example, R117H and I148T mutations may produce a more variable clinical phenotype, depending upon genetic modifiers, some of which may not be well defined. In addition, analytic errors are likely to be rare (i.e., 1:1000 to 1:10,000), particularly when confirmatory testing is performed.

Prevalence: The birth prevalence of $\mathrm{CF}$ varies by race/ ethnicity. Based on a literature review of prenatal screening trials, newborn screening trials, and systematic registries, the birth prevalence of CF in Caucasians is about 1:2500 (carrier rate 1/25). Ashkenazi Jewish individuals have a risk that is similar to that in Caucasians. Fewer data are available for other racial/ethnic groups, and thus the estimates are less confident.

Clinical positive predictive value: In this setting, the clinical positive predictive value can be defined as the proportion of couples with positive test results who are at a $25 \%$ risk of hav- ing an affected child. This value can be computed by knowing the analytic and clinical sensitivity and specificity and prevalence of the disorder. The major CF mutations are expected to produce a CF clinical phenotype, so the clinical positive predictive value will be high (most carrier couples will have the $25 \%$ reproductive risk). Exceptions will occur, however, because of analytic false positives and because of variable expressivity of some CFTR mutations.

Clinical negative predictive value: In this setting, the clinical negative predictive value can be defined as the proportion of couples with negative test results who are not at a $25 \%$ risk of having an affected child. The clinical negative predictive value is high because the disorder is rare. Results that compromise negative predictive value occur because the panel cannot detect $19 \%$ of carrier couples and because of analytical errors. A reasonable estimate for clinical negative predictive value is $99.96 \%$ ( 1 in 2500 negative couples are actually at a $25 \%$ risk compared with 1 in 625 prior to testing) (see Table 1).

CF 2.11.3 Test validation requirements: The laboratory must satisfy the test validation criteria described by ACMG and any and all state and federal applicable guidelines. Guidance is available from ACMG and other agencies, including New York State Department of Health (www.wadsworth.org), National Committee for Clinical Laboratory Standards (NCCLS, MM1-A Vol. 20, No. 7), and College of American Pathologists Checklist (www.cap.org).

CF 2.12 Diagnostic versus carrier testing: This test is used for confirmative diagnosis and carrier detection. Positive results are considered diagnostic rather than predictive, since penetrance is virtually $100 \%$. Carriers are asymptomatic. However, carrier screening may identify individuals who carry two mutations in the CFTR gene but are asymptomatic or present with nonclassic, mild symptoms or have a late-onset presentation. There are no reports of de novo mutations in CF. A larger number of mutations $(>25)$ is generally appropriate for diagnostic testing in order to achieve the highest possible clinical sensitivity.

CF 2.12.1.1 Prenatal testing: CF mutation analysis can be used for prenatal diagnosis in both direct and cultured amniotic fluid cells and chorionic villus samples. It is recommended that both parents be tested prior to testing of fetal specimens, preferably within the same laboratory. As appropriate, parents and fetus should be tested (or retested) within the same laboratory. The laboratory must specify the amount of material required for testing and provide referring professionals with appropriate instructions. Laboratories must have a prenatal follow-up program in place.

CF 2.12.1.2 Indications for prenatal testing: known parental mutations, family history of CF, echogenic bowel at ultrasound during the second trimester.

CF 2.12.1.3 Maternal cell contamination: All prenatal samples should be examined in parallel with a maternal sample to rule out error due to maternal cell contamination. A combination of several polymorphic STR sites is recommended. Sensitivity studies should be included in the assay validation to de- 
termine the acceptable detection level of maternal cell contamination.

\section{CF 3 GUIDELINES}

\section{CF 3.1 Pretest considerations}

CF 3.1.1 Informed consent is recommended for CF testing. It is the duty of the health care professional, not the laboratory, to obtain informed consent. It is the laboratory's responsibility to explain CF testing to the health care provider such that meaningful informed consent may be obtained.

CF 3.1.2 Laboratories should have a mechanism to collect pretest clinical information that includes patient's date of birth, indication for testing (see Section 2.9 above), racial/ethnic background, and specific family history of CF. If the patient has a positive family history, the laboratory should determine whether the familial mutation(s) is (are) known. Pretest information can be solicited using a specialized test requisition or questionnaire. The physician should be contacted if the preanalytical information does not accompany the specimen. If the laboratory is unable to obtain this information, the written report should contain language or tabular information to assist clients in interpreting the results. For example, a report for carrier risk revision may contain tables that allow the ordering physician to interpret carrier studies with negative finding, tabulated by ethnicity and family history. If the laboratory determines that the requested test is inappropriate, the ordering physician should be contacted immediately.

\section{CF 3.2 METHODLOGICAL CONSIDERATIONS}

All general guidelines for polymerase chain reaction (PCR) in the ACMG Standards and Guidelines apply. The following additional details are specific for CF. For this test, there are many valid methods with different strengths and weaknesses. A comparison of the methods described in this section is shown in Table 2.

CF 3.2.1 Positive controls: Many of the mutation-positive controls can be obtained from the NIGMS Human Genetic Cell Repository (http://locus.umdnj.edu/nigms/) as either cell lines or DNA. However, many mutations are not commercially available, which presents a problem for the laboratory validating their test. For mutations that are unavailable commercially, one option for the laboratory is to produce synthetic controls using PCR. All synthetic controls produced in the laboratory, however, must be validated by sequence analysis in both forward and reverse directions, to confirm the specific mutation. The amount used should be empirically determined and ideally be less than expected from a genomic sample. It is recommended that once the laboratory identifies a patient positive for such a mutation and provided that the patient has previously consented for re-use of his/her DNA, that genomic DNA be used as a positive control in future CF assays. If positive controls are generated with PCR, it is important that the laboratory take appropriate precautions to avoid contamination of patient assays with control PCR product. Although it is desirable that all positive controls be included in each assay, given
Table 2

Comparison of SNP technologies for CF tests

\begin{tabular}{|c|c|c|}
\hline & Strength & Weakness \\
\hline ASO & $\begin{array}{l}\text { High throughput potential } \\
\text { Potentially automatable } \\
\text { Easy to add mutations } \\
\text { Analytic validity/performance } \\
\text { characteristics published } \\
\text { Test simultaneously for a high } \\
\text { mutation spectrum }\end{array}$ & $\begin{array}{l}\text { Complex design } \\
\text { No commercial ASRs }\end{array}$ \\
\hline \multirow[t]{3}{*}{ RDB } & $\begin{array}{l}\text { Medium throughput } \\
\text { Commercially available ASRs }\end{array}$ & $\begin{array}{l}\text { Complex design } \\
\text { Difficult to add mutations }\end{array}$ \\
\hline & $\begin{array}{l}\text { Commercial development of } \\
\text { hybridization/reading } \\
\text { automation }\end{array}$ & $\begin{array}{l}\text { Not feasible to run all mutation } \\
\text { controls }\end{array}$ \\
\hline & Nonisotopic & \\
\hline ARMS & $\begin{array}{l}\text { One-few tube reactions } \\
\text { Commercial ASRs available } \\
\text { Nonisotopic } \\
\text { Rapid } \\
\text { Analytic validity/performance } \\
\text { characteristics published }\end{array}$ & $\begin{array}{l}\text { Complex design } \\
\text { Low throughput } \\
\text { Not feasible to run all mutation } \\
\text { controls } \\
\text { May not genotype unless paired } \\
\text { wild-type/mutation reactions } \\
\text { are done } \\
\text { Difficult conditions for each } \\
\text { mutation } \\
\text { Gel-based } \\
\text { Difficult to add mutations }\end{array}$ \\
\hline OLA & $\begin{array}{l}\text { Commercial ASRs } \\
\text { Nonisotopic }\end{array}$ & $\begin{array}{l}\text { Requires expensive equipment } \\
\text { Not feasible to run all mutation } \\
\text { controls }\end{array}$ \\
\hline
\end{tabular}

the large number of CF mutations in the standard test, it is not always practical to run all positive controls on every assay, particularly depending upon the laboratory and the specific technology used. At a minimum, during routine testing, it is recommended that each run include at least one positive assay control and that all positive controls be tested on a rotating basis. Thus, in each specific technology section, we address the issue of positive controls.

CF 3.2.2 Sample preparation: Multiplex PCR detection is amenable to the use of DNA prepared from blood by using a variety of extraction protocols, ranging from crude lysates to highly purified DNA depending on the sizes of the amplicons. This procedure also accommodates DNA prepared from buccal samples (i.e., brushes, swabs, and mouthwashings). It is recommended that DNA from prenatal samples (i.e., amniocytes and chorionic villi) be highly purified in order to be sufficient in quality and quantity for any additional testing that may be required. Typically, 10 to $50 \mathrm{ng}$ of patient DNA is adequate for a robust amplification reaction.

CF 3.2.3 Validation of methods: For CF mutation analysis, laboratories can currently choose between development of home-brew methodologies or use commercial analyte-specific reagents (ASRs). Laboratories developing genetic tests for clinical use under "home-brew" regulations are regulated only under the provisions of CLIA '88. CLIA ' 88 requires collection of in-house data to validate test performance prior to reporting results, but provides little detailed guidance that is relevant to 
DNA-based testing. Whether the laboratory chooses to develop CF testing as home-brew or use commercially available ASR reagents, it is the laboratory's responsibility to validate assay performance and to provide other information such as intended use of the test, methodology, and reporting formats. The State of New York Department of Health currently provides a helpful checklist for the preparation of genetic testing validation packages, and other guidelines are under review. For additional information on test validation procedures, refer to the ACMG Standards and Guidelines for Clinical Genetics Laboratories, 2002, Section C8 (http://www.ACMG.net).

\section{CF 3.2.3.1 Forward allele-specific oligonucleotide (ASO)}

\section{CF 3.2.3.1.1 Overview}

The ASO method is based upon hybridization of a labeled oligonucleotide probe containing either wild-type sequence or known mutant sequence to the target, patient DNA. This method has been described and applied to high-spectrum CF mutation analysis in a clinical laboratory setting. ${ }^{17-19}$ Generally, PCR products from multiplex PCR reactions of patient DNAs are manually or robotically spotted onto replicate filters (dot blots) and then hybridized to labeled ASOs under specific conditions. Design of the multiplex PCR conditions, ASOs, hybridization and wash conditions, and detection is complex. There are no commercial "kits" or ASRs currently available. An advantage of this method is that mutations can be readily added to an already existing panel. There are a number of issues that must be considered in the development of this test platform.

\section{CF 3.2.3.1.2 Design and labeling of ASO probes}

ASOs for the normal and mutant sequence pair should be derived from the same DNA strand. Since G:T and G:A mismatches are less destabilizing during hybridization reactions, it is important to avoid a G:T or G:A mismatch between the mutant oligonucleotide and the normal template. ASO probes are labeled for radioactive or chemiluminescent detection. If radioactively labeled, the laboratory determines the need for purification and quantification prior to use.

\section{CF 3.2.3.1.3 Multiplex PCR amplification}

Various parameters can be employed which allow the use of one PCR program for a combination of primer sets. One method is touchdown annealing cycling. Others may depend on primer design.

\section{CF 3.2.3.1.4 Dot-blot membranes}

To prepare replicate filters, the use a robotic system or a multichannel pipetting device is recommended to ensure that the same patient PCR product is placed at the same position on each filter. This is critical to the interpretation of the results of this assay.

\section{CF 3.2.3.1.5 Hybridization}

For radioactively labeled probes, it is recommended that an optimized and constant number of $\mathrm{cpm} / \mathrm{mL}$ be consistently used from run to run in order to obtain consistent quality of results. In addition, it is recommended that a nonlabeled competitive probe be included at an increased molar concentration (about 10- to 20-fold higher) in order to eliminate nonspecific signal (i.e., increase signal-to-noise ratio). The optimum conditions for hybridization must be determined by the laboratory. Optimal pooling strategies for combining probes should be determined by the laboratory if pooling is performed. Calculation of Tm for each oligonucleotide is insufficient to predict the correct conditions for hybridization, which must be empirically determined. Protocols describing a pooled hybridization condition have been described. ${ }^{17-19}$

\section{CF 3.2.3.1.6 Interpretation of results}

Comparison of the autoradiograph of the wild-type filter and the mutant filter based upon position is necessary for interpretation of test results. In general, a positive result at a given position only on the wild-type filter is interpreted as normal, a positive only on the mutant filter is interpreted as homozygous for the mutation, and a positive on both filters is interpreted as heterozygous for the mutation. For CF analysis, a number of filters is necessary to obtain the minimum panel of the 25 recommended mutations. Thus it is important that results from all filters be read prior to interpretation, particularly when two different mutations are detected in the same patient, such as in diagnostic testing. A grid placed over the filters is recommended for location of exact position, particularly when the analysis is performed in a 96-well format. It is also recommended that at least two (or more) individuals read the results and concur prior to reporting.

\section{CF 3.2.3.1.7 Reflex testing}

Rare DNA variants can cause failure of amplification or failure of the ASO to hybridize. Of particular concern is the presence of apparent homozygosity for the $\Delta$ F508 mutation by ASO analysis. It is critical that laboratories include known variants in the mutation panel to prevent mistyping of compound genotypes such as F508C/ $\Delta$ F508. Laboratories may wish to confirm all $\Delta$ F508 homozygous results, particularly unexpected homozygous results, by another type of analysis, such as gel analysis. Laboratories should be aware that failure of one allele to amplify can also lead to apparent homozygosity. In certain cases of unusual findings, such as homozygosity for rare mutations, laboratories may consider testing parents to confirm the genotype.

\section{CF 3.2.3.2 Reverse dot-blot (RDB) hybridization}

\section{CF 3.2.3.2.1 Overview}

An alternative approach to ASO analysis is RDB hybridization. In this method, the roles of the oligonucleotide probe and the target amplified DNA are reversed. Probe pairs, complementary to mutant and normal DNA sequences, are bound to nylon membranes in the form of dots or slots. DNA that has been amplified in multiplex reaction(s) and labeled using endlabeled primers or internal incorporation of biotinylated 
dUTP is hybridized to the membrane. This procedure is very amenable to high throughput analysis of high mutation spectrum genes and has been applied to the detection of $\beta$-thalassemia and CFTR mutations. ${ }^{20-22}$ Although probe design and production of the spotted membranes may be complex, mutation detection using this method is nonradioactive, convenient, rapid, and robust and requires no specialized interpretation skills. Commercial sets of ASRs are available, and sufficient published information exists so that laboratories can develop home-brew assays. Two colorimetric and one chemiluminescent biotin-based detection system have been reported. This technology, while robust, is relatively inflexible and not easily expanded to include additional mutations.

\section{CF 3.2.3.2.2 Oligonucleotide probe design}

Probes are conjugated at the $5^{\prime}$ end by an amino linker group, added by an aminophosphoramidite during synthesis, for subsequent covalent linkage to the carboxyl group of the activated nylon membrane. Length of the allele-specific primer and base composition must be optimized so that the final optimal hybridization and washing conditions for all detected alleles are identical. Probes with lengths 15 to 17 nucleotides with $30 \%$ to $50 \%$ GC content are adequate to discriminate point mutations. Otherwise, the same guidelines apply as for probe preparation for forward ASO hybridization. However, despite these general rules, probe design for adequate detection may also involve trial and error.

\section{CF 3.2.3.2.3 Strip layout, manufacture, and quality control}

Covalent linkage of the amino-modified oligonucleotide to the membrane-bound activated carboxyl group increases the sensitivity of the assay relative to previous enzymatic probe tailing methods. Each oligonucleotide solution should contain a dye such as phenol red to allow for visual inspection of the spotted membranes. The arrangement of oligonucleotides on the strip is a matter of personal preference; wild-type and mutant probes can be spotted in separate rows or groups, or interspersed among each other. Manual production of RDB strips is described by Cai et al. ${ }^{21}$; this process is amenable to robotic production of large strip lots that can then be stored at room temperature until use. Each lot of strips should be compared to a previous lot to verify consistency with respect to each allele detected in the assay as well as a negative (no DNA) control. For home-brew strip production, it is often necessary to adjust the amount of new lots of probe that is applied to the strips in order to optimize hybridization signal.

\section{CF 3.2.3.2.4 Multiplex PCR amplification}

All general guidelines for multiplex PCR amplification apply to RDB detection. It has been reported that semi-nested PCR may increase hybridization signal for some mutations. ${ }^{22}$ It is useful to design the primers so that each product differs by at least $10 \mathrm{bp}$ in length so that robustness of amplification can be visualized on a check gel prior to hybridization. The choice of probe labeling depends on the detection system; primers are biotinylated at the $5^{\prime}$ terminus for subsequent strepavidinhorseradish peroxidase detection.

\section{CF 3.2.3.2.5 Controls}

While the laboratory may determine that it is not feasible to include each positive assay control in each run due to batch size limitations, QC on a new lot of RDB should include testing for each mutation. At a minimum, during routine testing, it is recommended that each run include at least one positive assay control and that all positive controls be tested on a rotating basis. The number of positive controls can also be minimized by using genomic or synthetic compound heterozygotes.

\section{CF 3.2.3.2.6 Hybridization, detection and interpretation}

Hybridization and detection are straightforward and require minimal labor. Care should be taken to protect lightsensitive reagents. The genotype of the patient is easily read from the array of hybridization signal on each strip. Individual test results should be read by two reviewers and concur prior to reporting. Since the hybridization signal fades over time, the strips should be photocopied, photographed, digitized, or scanned in order to keep a permanent result record for each patient.

\section{CF 3.2.3.2.7 Reflex testing}

One of the strengths of the RDB method is the ability to test simultaneously for a high mutation spectrum. However, additional labor is incurred when mutations are tested only as a reflex. As for ASO typing, it is critical to include frequent polymorphisms in the coding region of the CFTR gene, e.g., F508C, to prevent mistypings of polymorphism/mutant compound heterozygous genotypes such as $\mathrm{F} 508 \mathrm{C} / \Delta \mathrm{F} 508$. As described below, it is desirable to determine the 5/7/9T genotype only for diagnostic cases or carriers positive for the $\mathrm{R} 117 \mathrm{H}$ mutation. However, commercial versions using RDB my not offer this option, since all of the alleles, including the 5/7/9T probes, are contained on a single strip. If these data are generated, it should be reported, albeit with caution, so that patients are not unduly alarmed.

\section{CF 3.2.3.3 Amplification refractory mutation system (ARMS)}

\section{CF 3.2.3.3.1 Overview}

ARMS, or allele specific amplification, is the PCR equivalent of allele-specific hybridization with ASO probes. Worldwide, ARMS is one of the most frequently used methods for multiplex detection of common CFTR mutations, partly due to the commercial availability of kits and ASR reagents. Advantages of the ARMS method are that it is rapid (results can be obtained in one working day), reliable, and nonisotopic. In addition, analytic validity and other performance characteristics of ARMS for the specific application of CF carrier testing can be estimated by using data from eight published reports. ${ }^{23-30}$ Most of these studies used primers obtained from the same commercial source. 
PCR reactions depend on two oligonucleotide primers that bind to the complementary strands at either end of the DNA segment to be amplified. ARMS is based on the observation that oligonucleotide primers that are complementary to a given DNA sequence except for a mismatch (typically at the 3' $\mathrm{OH}$ residue) will not, under appropriate conditions, function as primers in a PCR reaction. For genotyping, paired PCR reactions are performed for each mutation tested. One primer (common primer) is used in both reactions, while the other is either specific for the mutant or wild-type sequence. In principle, ARMS tests can be developed for any single base pair change or small deletions/insertions. Achieving acceptable specificity is dependent on primer selection and concentration. Use of longer primers (e.g., $30 \mathrm{vs.} 20 \mathrm{bp}$ ) and inclusion of control reactions have been reported to improve specificity. Primers and conditions for multiplex reactions must be selected so that the relative yields of PCR products are balanced and the PCR products can be adequately separated on agarose gels. Detection of 25 mutations is likely to require two or more multiplex reactions.

Home-brew primer sets must be validated to ensure desired performance characteristics, and new reagent lots should be compared to a previous lot to ensure consistency in performance and robustness. One commercial set of ASR ARMS reagents for detecting 29 CF mutations is available in the United States. Although the manufacturer performs a level of performance evaluation on these reagents, the laboratory must also complete an internal validation to assess proficiency prior to use on patient samples.

\section{CF 3.2.3.3.2 Controls}

Internal control reactions are not required if mutant and wild-type ARMS reactions are combined in the same test. However, for screening purposes, multiplexing mutant ARMS reactions without paired wild-type reactions can result in significant cost savings. Internal controls (additional control primers that amplify unrelated sequences) can be included in each multiplex reaction to ensure that DNA samples will generate at least one PCR product in each tube and reduce the likelihood of false-negative results. Negative and positive control samples must be run with each assay, but the laboratory may determine that it is not feasible to include all 25 mutation controls in each run due to batch size limitations. Pooled positive DNA control samples can be utilized to allow efficient inclusion of the most common mutation controls in each run. Remaining positive controls can be tested on a rotating basis.

\section{CF 3.2.3.3.3 Visualization and interpretation of results}

PCR products are separated by electrophoresis through an agarose gel containing ethidium bromide and visualized by UV transillumination. Individual test results are interpreted by analysis of the banding pattern by two reviewers in comparison with a molecular weight standard. Assays without paired wildtype reactions (including the available ASR reagents) are effective for carrier testing, but will not identify rare homozygotes for mutations other than $\Delta$ F508 ( $2 \%-3 \%$ of CF cases).

\section{CF 3.2.3.4 Oligonucleotide ligation assay (OLA)}

CF 3.2.3.4.1 Overview

The OLA is a novel approach to mutation detection of point mutations, small deletions, and small insertions, and it consists of two phases. The first reaction, allele-specific PCR, based upon hybridization of an exact-match PCR primer to the target sequence, is performed. PCR primers are designed with either the normal or mutant nucleotide(s) at the ultimate $3^{\prime}$ end and a differential mobility modifier sequence used to distinguish various PCR products based on size at the 5 ' end. The second reaction is a ligation reaction in which the amplified product from the first PCR reaction is ligated to a common probe, an ASO consisting of sequences immediately adjacent to the mutation site. The common probe is phosphorylated at the $5^{\prime}$ end to allow for the ligation reaction and contains a fluorescent dye marker at the 3 ' end to allow detection upon separation. Detection requires the use of an automated sequencer capable of multifluorescence detection, and it may be performed in a gel or capillary format. The normal and mutant peaks are identified on the basis of their product size and fluorescent tag. A properly designed OLA gives only the appropriate normal or mutant product(s). A CF OLA kit is commercially available. The kit contains all reagents necessary to perform testing for $31 \mathrm{CF}$ mutations as well as the normal counterparts and has been described. ${ }^{31}$ The entire analysis is performed in a single tube. In addition, the kit includes a specific template that is necessary for interpretation of results. The template is intended for use with commercially available software to analyze data and create summary reports. While the manufacturer has performed validation studies for this kit, it is also important that the laboratory perform a minimal internal validation to assess proficiency prior to use on patient samples. See Section 3.2.2.

\section{CF 3.2.3.4.2 Controls}

If practical for the laboratory, it is desirable to include all positive controls in each assay. However, it may not be feasible to include numerous positive controls in each assay run. Minimally, negative controls, a heterozygous and homozygous positive control for $\Delta \mathrm{F} 508$, and several "no DNA" controls should be included in each run. Additional positive controls should be rotated among assay runs. The assay should be robust and give consistent results with relative peak heights and mutation assignment. While the software automatically interprets the data, it is important that the results be confirmed visually by the laboratory director or designee.

\section{CF 3.2.3.4.3 Visualization and interpretation of results}

Fluorescently labeled PCR products are separated by electrophoresis using an automated sequencer, either gel or capillary-based. The data are analyzed with commercially available software, and interpretation of results is achieved by comparison to a CF template. Wild-type controls will be homozygous for the normal product, appearing as a single peak migrating at the position of the normal sequence. Heterozygotes will appear 
as two peaks, both wild-type and mutant, but at half-intensity in height. Homozygous mutant samples will appear as only one peak that migrates in the position of the mutant sequence. Since as many as 31 mutations can be analyzed simultaneously in one reaction tube, it is critical that the position of migration for each allele be appropriately validated to ensure accurate interpretation of patient results. It is also important that the laboratory set thresholds for peak height to avoid pitfalls of misinterpretation due to background noise. It is recommended that the laboratory verify that the multiplex reaction, which includes all alleles to be analyzed, both normal and mutant, is robust and reproducible. Automated peak assignment is an attractive feature of the software, which is desirable for quality assurance issues. Visual inspection, however, is recommended.

CF 3.2.3.5 Additional methods for performing high mutation spectrum and high throughput SNP analyses exist, although few are currently in use in clinical molecular genetic laboratories in the United States. These methods (which are not all-inclusive) include flow cytometry-based detection of bead-coupled ASOs, various arrayed primer extension methods, mass spectrometry detection methods, oligonucleotide array approaches, and minisequencing of target regions. However, at the present time, most (but not all) of these technologies are severely limited in ability to perform multiplex analysis. Thus, while they may be applicable for use in testing for a small number of variants, such as hemochromatosis or factor V Leiden, they currently have not been applied to the detection of a large number of mutations as required in the CF analysis. We anticipate that future improvements in these technologies or others will make them adaptable to CF analysis. Thus they will be included in these guidelines at a later date.

\section{CF 3.2.4 Guidelines for development of primers and probes}

General considerations include sequence composition, Tm, GC content, size of desired product, intron/exon boundary inclusions to detect splice-site mutations, and avoidance of polymorphisms at the primer site. Any home-brew primer sets should be thoroughly tested to ensure desired performance characteristics.

CF 3.2.4.1 Published lists of primers are available. ${ }^{8,32}$ Several sets of primers, PCR conditions, and methods of separation and detection have been published.9,17 Other primers and methods can be used if adequate validation is performed.

CF 3.2.4.2 Home-brew primers can be developed using any commercially available primer design software package that helps to select optimum sets of primers based on Tm and salt concentration.

CF 3.2.4.3 Multiplex considerations: General issues to consider in designing a multiplex PCR analysis include optimum design of several sets of primers for amplification under a single set of conditions including the same Tm, length of primer, compatibility of primers (avoidance of primer interactions), specificity of primers, avoidance of pseudogenes and known polymorphisms, similar GC content, optimizing salt concen- tration, determining concentration of each primer to use in reaction (trial and error), unifying the annealing temperature by using commercially available buffers (such as $\mathrm{Q}$ solution) or DMSO, ${ }^{33}$ and type of Taq (i.e., Taq Gold, Hot-start, etc). Generally, for multiplex PCR reactions, lower primer concentrations are recommended and higher dNTP concentrations are required. For the $\mathrm{CF}$ gene, the 25 recommended mutations are found in 15 exons (or intronic regions); thus a 15-plex reaction would be required for amplification of all in a single tube. For the complete CF gene analysis including 27 exons, the laboratory may perform multiple multiplex PCR reactions. It is the laboratory's responsibility to validate all assays in which PCR primers are designed in-house. For troubleshooting assay failures it is recommended that multiplex assays be designed with each PCR product of a different length and sufficient to visualize on an agarose gel to determine the presence and amount of product. Commercial PCR optimizing kits are available to aid laboratories in development efforts.

CF 3.2.4.4 Setting optimum reaction conditions: Factors to consider include optimization of salt concentration and primer concentration, choice of buffer, and choice of Taq. Single PCR reactions will have different reaction conditions from multiplex PCR reactions. It is important to set these conditions to obtain a robust PCR product reproducibly, yet to avoid spurious results.

CF 3.2.4.5 Setting optimum cycling conditions: Various approaches exist in setting these conditions. Step-down conditions have been described and are particularly useful for multiplex reactions when primers anneal at various temperatures. ${ }^{17}$ Generally, cycling conditions should include no more than 35 cycles in order to avoid introduction of errors. The cycling conditions should be set for high stringency to obtain pure products. Annealing temperature should be closely determined by $\mathrm{Tm}$ of primers. It is advisable for the laboratory to develop primers that use the same set of reactions and cycling conditions. After PCR the laboratory may or may not choose to examine the PCR product on an agarose gel.

\section{CF 3.2.4.6 General disclaimer about primer-binding/probe annealing regions}

It should be realized that there are many sources of diagnostic errors. Genotyping errors can result from trace contamination of $P C R$ reactions and from rare genetic variants that interfere with analysis. Additionally, polymorphisms in targeted regions (primer-binding or probe-annealing) can lead to testing errors and result in failure of one allele to amplify (allele drop-out).

\section{CF 3.3 Mutation panel}

CF 3.3.1 Minimum mutation panel for population-based carrier screening purposes ${ }^{1}$ : Different testing panels might be used for identification of CFTR mutations in patients with diagnosed CF, in relatives of patients with CF, or in newborn screening. It is important to recognize that this panel is subject to change as new information becomes available. Conse- 
quently, with the emergence of a vast amount of new data from multiple laboratories using this initial mutation panel, data evaluation is in progress with the goal of developing a revised mutation panel in 2002.

$\begin{array}{lllll}\Delta \text { F508 } & \text { R553X } & \text { R1162X } & \text { 2184delA } & 3120+1 \mathrm{G}>\mathrm{A} \\ \Delta \mathrm{I} 507 & \mathrm{G} 542 \mathrm{X} & \mathrm{G} 551 \mathrm{D} & \mathrm{W} 1282 \mathrm{X} & \text { N1303K } \\ 621+1 \mathrm{G}>\mathrm{T} & \text { R117H } & 1717-1 \mathrm{G}>\mathrm{A} & \text { A455E } & \text { R560T } \\ \text { G85E } & \text { R334W } & \text { R347P } & 711+1 \mathrm{G}>\mathrm{T} & 1898+1 \mathrm{G}>\mathrm{A} \\ 1078 \text { delT } & 3849+10 \mathrm{kbC}>\mathrm{T} & 2789+5 \mathrm{G}>\mathrm{A} & 3659 \text { delC } & \text { I148T }\end{array}$

CF 3.3.2 Inclusion of the common R117H mutation in the test panel screens for CBAVD as well as for CF: The phenotypic consequences of the $\mathrm{R} 117 \mathrm{H}$ mutation are modulated in cis by the 5/7/9T polypyrimidine tract in intron 8 such that $\mathrm{R} 117 \mathrm{H} / 7 \mathrm{~T}$ is associated with CBAVD and $\mathrm{R} 117 \mathrm{H} / 5 \mathrm{~T}$ is associated with CF. ${ }^{34}$ Moreover, the $5 \mathrm{~T}$ allele is associated as a trans mutation in CBAVD. ${ }^{35}$ It is recommended that the 5/7/9T variant be excluded from the routine carrier screen but tested as a reflex for carriers shown to be heterozygous for the $\mathrm{R} 117 \mathrm{H}$ mutation. The 5/7/9T variant should be included for diagnostic panels to distinguish the genotypes of $\mathrm{R} 117 \mathrm{H}$ associated with CF from those associated with CBAVD and as a potential pathogenic mutation for CBAVD. As described above, if it is not feasible to test the 5/7/9T variant as a reflex for technologies such as RDB, this variant should be reported to avoid compliance issues.

CF 3.3.3 Issues of unexpected homozygosity due to polymorphisms: Tests may not distinguish between a CF mutation and benign variants. For example, I506 V and F508C are performed as reflex tests for $\Delta \mathrm{F} 508$ positives unless it is proven that these variants do not cause assay interference.

CF 3.3.3.1 Incorrect assignment of homozygosity: Deletions, polymorphisms, and benign variants can lead to incorrect assignment of homozygosity when a benign variant is present at the same site on the second allele. Parental testing to confirm homozygosity is recommended for rare mutations.

CF 3.3.4 Controls representing the mutations to be tested should be run on each assay, if feasible, based upon the testing method. Laboratories should validate their control DNA by sequencing, by exchange with another laboratory, or by using consensus-validated material.

CF 3.3.5 Laboratories that service a particular ethnic population based on geography may consider including additional mutations in the testing panel that are specific to that particular population. Every effort should be made to determine the frequency of specific CF mutations within the target population and to provide testing at reasonably high sensitivity levels.

CF 3.3.6 An extended mutation panel may be appropriate for certain diagnostic testing purposes, but it is not recommended for routine carrier screening of reproductive couples. If a laboratory offers an extended panel, it is important that the composition be determined on the basis of frequency of the mutation within the target population. The 25-mutation panel was based upon a $0.1 \%$ frequency worldwide. An extended panel would go beyond that requirement and expand its scope to the population of service, such as the U.S. population. Additional mutations of $>0.1 \%$ frequency in the U.S. population that laboratories may wish to consider adding to the minimum panel have been recently described. ${ }^{36}$

CF 3.3.7 Testing for unknown mutations using scanning technology/sequence analysis: All scanning methodologies described in the ACMG Standards and Guidelines for Clinical Genetics Laboratories apply.

Detection of a sequence alteration by a scanning technology must be confirmed by sequence analysis and interpreted according to the ACMG Recommendations for Standards for Interpretation of Sequence Variations (http://www.acmg.net). In addition, the alteration must be named according to the accepted guidelines for mutation nomenclature. The nomenclature developed by the Ad Hoc Committee on Mutation Nomenclature ${ }^{37}$ and Antonarakis et al..$^{38}$ is recommended. The nomenclature established for CFTR mutations follows these guidelines and is found in the CF mutation database at http://www.genet.sickkids.on.ca.

CF 3.3.8 Linkage analysis in CF families in which one or no CFTR mutations have been identified: Multiple informative markers are available within the CFTR gene and flanking the gene. It is recommended that more than one marker be included in the analysis and that the laboratory follow standard linkage analysis procedures in preanalytical, analytical, and postanalytical testing. The use of intragenic markers is preferred over the previously used extragenic markers. Prior to performing linkage analysis, it is recommended that the laboratory obtain confirmation of the clinical diagnosis of CF in the family.

CF 3.4 Quality assurance: Laboratories must follow the ACMG/CAP checklists and be in compliance with the NIHDOE Task Force on Genetic Testing (http://www.genome.gov/ page.cfm?page ID = 10001733) and follow the ACMG Standards and Guidelines for Clinical Genetics Laboratories. Laboratories must also participate in the CAP/ACMG Proficiency Testing Program or other interlaboratory proficiency testing program. All aspects of testing, including pre- and postanalytical, must be in full compliance with regard to appropriateness of test ordering, interpretation, reporting, and counseling. Laboratories must validate their CF assays, whether home-brew or commercial kit, and state the analytical and clinical sensitivity and specificity according to the ACMG guidelines.

\section{CF 3.5 Interpretations (postanalytical)}

CF 3.5.1 The following elements must be included in the report, in addition to the items described in the current general Standards and Guidelines:

CF 3.5.1.1 Ethnicity, indication for testing, test method with the FDA statement regarding the use of ASRs, test result, mutations tested, and residual risk based on ethnicity should be included.

CF 3.5.1.2 Clear interpretation of the patient result as homozygous for a mutation (predicted affected with CF), a compound heterozygote (predicted affected with CF), heterozygous carrier (interpretation depends on whether this is carrier testing or diagnostic testing), or negative (interpretation de- 
pends on whether this is carrier testing, presence or absence of family history, or diagnostic). In cases in which mutations have been identified, the mutation(s) name should be included. For examples of appropriate report components, laboratories should refer to the CF report templates for carrier screening as described by Grody et al. ${ }^{1}$

CF 3.5.1.3 All positive results for diagnostic tests or for positive/positive couple screening should state that genetic counseling is indicated and testing is appropriate for at-risk family members. When sequential carrier testing is done, a positive result on one partner should include the recommendation of testing the partner and at-risk family members. All individuals who have a family history of CF should receive genetic counseling. All CFTR carriers, including healthy males who have mutations associated with infertility, should also be referred for genetic counseling.

CF 3.5.2 Comments on phenotype issues with CBAVD, R117H and 5T, 7 T background: ACMG has recommended that all $\mathrm{R} 117 \mathrm{H}$ positive results require reflex testing for the $5 \mathrm{~T} / 7 \mathrm{~T} / 9 \mathrm{~T}$ variant in the polythymidine tract at intron 8 in CFTR gene. Refer to model reports for carrier screening presented in the ACMG statement. ${ }^{1}$ For R117H/5T positive heterozygotes, testing of parents is recommended to determine the inheritance of the $\mathrm{R} 117 \mathrm{H}$ and the $5 \mathrm{~T}$ variant (i.e., cis vs. trans position). If the $\mathrm{R} 117 \mathrm{H}$ and $5 \mathrm{~T}$ variant are determined to be in $c i s$, then the report should reflect that this mutation has been associated with a variable phenotype when $\mathrm{R} 117 \mathrm{H} / 5 \mathrm{~T}$ (cis) or another CFTR mutation is present in patients with CF. If the $\mathrm{R} 117 \mathrm{H}$ mutation and $5 \mathrm{~T}$ are determined to be in trans, the report should indicate that the individual carries a relatively benign CF mutation that is not generally associated with the phenotype of typical CF patients but has been associated with CBAVD, leading to infertility in males and no known clinical features in females. In addition, the report should reflect that the $5 \mathrm{~T}$ variant on one chromosome, in combination with a CFTR mutation on the opposite chromosome, may lead to male infertility due to CBAVD, with or without mild or atypical symptoms of CF, and that there is no known clinical significance of $5 \mathrm{~T}$ in females. The penetrance of $5 \mathrm{~T}$ is reduced, and thus it is difficult to predict the clinical significance of the $5 \mathrm{~T}$ variant. For individuals who are $\mathrm{R} 117 \mathrm{H}$ positive and $5 \mathrm{~T}$ negative, the report should indicate that the $\mathrm{R} 117 \mathrm{H}$ mutation is not expected to lead to a typical CF clinical phenotype. However, R117H has been associated with CBAVD. In all above cases, genetic counseling is recommended. For diagnostic testing, and particularly for testing for CBAVD in males with infertility, it is recommended that the intron 8 variant be included in the testing panel.

CF 3.5.3 Comments on individual residual risk and reproductive risk for couples should be included in the patient report or provided to the referring health care professional. Table 1 is given as an example to be helpful for the laboratory and is not intended to be all-inclusive of every ethnic group. Several assumptions were used in developing the risk values in this table, including carrier frequencies of various racial/ethnic groups and sensitivities of the minimum mutation panel of 25 muta- tions in these various populations. This table is intended for use in CF screening of reproductive couples who have no family history of CF. For individuals with a family history of CF, the calculations would be different and would be based upon pedigree information. It is the laboratory's responsibility to provide this type of information, specific for the population it serves.

CF 3.5.4 Residual risk for fetus with echogenic bowel: Echogenic bowel in the fetus based upon ultrasound, present in $0.1 \%$ of all pregnancies, can be due to CF or may be associated with normal variation, chromosome abnormality, or congenital viral infection. ${ }^{39}$ There have been relatively few studies to determine the frequency with which echogenic bowel in the fetus correlates with CF. Thus it is difficult to determine a prior risk when echogenic bowel is identified in a second-trimester fetus upon ultrasound. Collective data suggest a risk of approximately $1 \%$, which has been used in calculating posterior $\mathrm{CF}$ risk in a fetus with echogenic bowel and heterozygous for a $\mathrm{CF}$ mutation. ${ }^{39-42}$ There are two publications describing echogenic bowel calculations, one using Bayesian analysis ${ }^{43}$ and one using a complex probability calculation ${ }^{42}$ which laboratories may use. In calculating risk, carrier frequency and the test sensitivity in the specific racial/ethnic population must be considered. Whether or not to provide residual risk information for these cases is left to the laboratory's discretion, as the literature is limited and additional data collection is desirable to provide accuracy in risk assessment. Some laboratories, however, will take the view that even limited information can be useful for these families. For such laboratories, we provide the following information.

\section{CF 3.5.4.1 Example of a laboratory report for a fetus with echogenic bowel}

It is important to recognize that there is considerable heterogeneity in ultrasound finding reported by different examiners. This report addresses the situation of typical echogenic bowel in the second trimester. It should be recognized that calcifications in the liver and findings suggestive of peritonitis in the third trimester are significantly different. There is a published report ${ }^{40}$ indicating that there may be an empirical risk that $13 \%$ of such fetuses prove to have CF. This number may in fact be as low as 3\% (Baylor DNA Diagnostic Laboratory, unpublished data). Calculations can be made using a range of empirical risk for these fetuses between $3 \%$ and $13 \%$. Obviously, if the fetus has two CF mutations, this is diagnostic of CF. A fetus with echogenic bowel and one identified CF mutation represents the most difficult counseling circumstance. The risk of such a fetus to be affected with CF can be calculated to be within a range of $13 \%$ to $43 \%$, depending upon the assumption regarding the prior empirical risk. If no mutation is detected in the fetus, the risk for the fetus to be affected with CF would be equal to or $<1$ in 645 . These risks are calculated on the basis of the assumption of a Caucasian fetus of Northern European ancestry, a carrier frequency of 1 in 25, and a test sensitivity of $90 \%$. The calculations would be different for a fetus of Ashkenazi Jewish, African-American, Hispanic, or 
other ethnic background, based on differences in the test sensitivities and the carrier frequencies for each of these populations. It is also important to consider other pathology in such cases such as chromosome abnormality, intestinal malformation, or congenital infection (particularly if calcification is present).

\section{CF 4 POLICY STATEMENTS}

CF 4.1 The NIH Consensus Conference ${ }^{7}$ issued a statement that CF mutation testing should be made available to all pregnant couples.

CF 4.2 The ACMG issued a policy statement titled "Laboratory Standards and Guidelines for Population-Based Cystic Fibrosis Carrier Screening."' This document is also available online at http://www.acmg.net.

CF 4.3 The American College of Obstetricians and Gynecologists, in collaboration with ACMG and the National Human Genome Research Institute, have developed and distributed clinical and laboratory guidelines (October 2001). One document titled "Preconception and Prenatal Carrier Screening for Cystic Fibrosis: Clinical and Laboratory Guidelines" provides information for providers. Two patient educational brochures titled "Cystic Fibrosis Carrier Testing: The Decision is Yours" and "Cystic Fibrosis Testing: What Happens If Both My Partner and I Are Carriers?" were developed to help patients with their decisions (http//www.acog.org).

Approved by the Board of Directors of the American College of Medical Genetics, January 26, 2002.

\section{(c) AMERICAN COLLEGE OF MEDICAL GENETICS 2002}

\section{Acknowledgments}

The authors offer sincere thanks to Dr. Larry Silverman for helpful discussions and review of the final manuscript. We also thank Dr. Madhuri Hegde for providing helpful information and discussions regarding the use of OLA for CF analysis, the Baylor DNA Diagnostic Laboratory for use of their report as an example, and Mr. Glenn Palomaki for critical review and contribution on the clinical validation section. We wish to thank the additional members of the Laboratory Quality Assurance Committee for their careful review of this document, including Drs. Arthur Brothman, Daynna Wolff, Peter Jacky, Betsy Hirsch, Geraldine McDowell, Robert Grier, and Robert Desnick.

\section{References}

1. Grody WW, Cutting GR, Klinger KW, Richards CS, Watson MS, Desnick RJ. Laboratory standards and guidelines for population-based cystic fibrosis carrier screening. Genet Med 2001;3:149-154.

2. Kerem B-S, Rommens JM, Buchanan JA, Markiewicz D, Cox TK, Chakravarti A, Buchwald M, Tsui LC. Identification of the cystic fibrosis gene: genetic analysis. Science 1989;245:1073-1080 (erratum 1989;245:1437).

3. Riordin JR, Rommens JM, Kerem B-S, Alon N, Rozmahel R, Grelczak Z, Zielenski J, Lok S, Plavsic N, Chou JL. Identification of the cystic fibrosis gene: cloning and characterization of complementary DNA. Science 1989;245:1066-1073.
4. Rommens JM, Iannuzzi MC, Kerem B-S, Drumm ML, Melmer G, Dean M, Rozmahel R, Cole JL, Kennedy D, Hidaka N. Identification of the cystic fibrosis gene: chromosome walking and jumping. Science 1989;245:1059-1065.

5. Cystic Fibrosis Genetic Analysis Consortium. Population variation of common cystic fibrosis mutations. Hum Mutat 1994;4:167-177.

6. Cystic Fibrosis Foundation. Cystic Fibrosis Foundation Patient Registry Annual Data Report, 1998. Bethesda, MD: Cystic Fibrosis Foundation.

7. NIH Consensus Development Conference Statement. Genetic testing for cystic fibrosis. April 14-16, 1997. Arch Intern Med 1999;159:1529-1539.

8. Abeliovich D, Lavon IP, Lerer I, Cohen T, Springer C, Avital A, Cutting GR. Screening for five mutations detects $97 \%$ of cystic fibrosis (CF) chromosomes and predicts a carrier frequency of 1:29 in the Jewish Ashkenazi population. Am J Hum Genet 1992;51:951-956.

9. DeMarchi JM, Caskey CT, Richards CS. Screening for diseases frequent in the Ashkenazi Jewish population. Hum Mutat 1996;8:116-125.

10. Eng CM, Schechter C, Robinwitz J, Fulop G, Burgert T, Levy B, Zinberg R, Desnick RJ. Prenatal genetic carrier testing using triple disease screening. JAMA 1997;278: 1284-1285.

11. Orgad S, Neumann S, Loewenthal R, Netanelox-Shapira I, Gazit E. Prevalence of cystic fibrosis mutation in Israeli Jews. Genet Test 2001;5:47-52.

12. Macek M, Mackova A, Hamosh A, Hilman BC, Selden RF, Lucotte G, Friedman KJ, Knowles MR, Rosenstein BJ, Cutting GR. Identification of common cystic fibrosis mutations in African-Americans with cystic fibrosis increases the detection rate to 5\%. Am J Hum Genet 1997;60:1122-1127.

13. Grebe TA, Seltzer WK, DeMarchi J, Silva DK, Doane WW, Gozal D, Richter SF, Bowman CM, Norman RA, Rhodes SN et al. Genetic analysis of Hispanic individuals with cystic fibrosis. Am J Hum Genet 1994;54:443-446.

14. Arzimanoglou I, Tuchman A, Li Z, Gilbert F. Cystic fibrosis carrier screening in Hispanics. Am J Hum Genet 1995;56:544-547.

15. Villalobos-Torres C, Rojas-Martinez A, Villareal-Castellanos E, Cantu JM, SanchezAnzaldo FJ, Saiki RK, Barrera-Saldana HA. Analysis of 16 cystic fibrosis mutations in Mexican patients. Am J Med Genet 1997;69:380-382.

16. Palomaki GE, Haddow JE, Bradley LA, FitzSimmons SC. An updated assessment of cystic fibrosis mutation frequencies in non-Hispanic Caucasians. Genet Med 2002; 4:90-94.

17. DeMarchi JM, Richards CS, Fenwick RG, Beaudet AL. A robotics-assisted procedure for large-scale cystic fibrosis mutation analysis. Hum Mutat 1994;4:281-290.

18. Shuber AP, Skoletsky J, Stern R, Handelin B. Efficient 12-mutation testing in the CFTR gene: general model for complex mutation analysis. Hum Mol Genet 1993;2: 153-158.

19. Shuber AP, Michalowsky LA, Nass GS, Skoletsky J, Hire LM, Kotsopoulos SK, Phipps MF, Barberio DM, Klinger KW. High throughput parallel analysis of hundreds of patient samples for more than 100 mutations in multiple disease genes. Hum Mol Genet 1997;6:337-347.

20. Chehab FF, Wall J. Detection of multiple cystic fibrosis mutations by reverse dot blot hybridization: a technology for carrier screening. Hum Genet 1992;89:163-168.

21. Cai S, Wall J, Kan YW, Chehab FF. Reverse dot blot probes for the screening of $\beta$-thalassemia mutations in Asians and American blacks. Hum Mutat 1994;3:59-63.

22. Wall J, Cai S, Chehab FF. A 31-mutation assay for cystic fibrosis testing in the clinical molecular diagnostics laboratory. Hum Mutat 1995;5:333-338.

23. Feldmann D, Guittard C, Georges MD, Houdayer C, Magnier C, Claustres M, Couderc R. Genetic testing for cystic fibrosis: validation of the ELUCIGENE ${ }^{\mathrm{TM}}$ CF20 kit in blood and mouthwash samples. Ann Biol Clin 2001;59:277-283.

24. Bradley LA, Johnson DA, Chaparro CA, Robertson NH, Ferrie RM. A multiplex ARMS test for 10 cystic fibrosis (CF) mutations: evaluation in a prenatal CF screening program. Genet Test 1998;2:337-341.

25. Robertson NH, Weston SL, Kelly SJ, Duxbury NJ, Pearce SR, Elsmore P, Webb MB, Newton CR, Little S. Development and validation of a screening test for 12 common mutations of the cystic fibrosis CFTR gene. Eur Respir J 1998;12:477-482.

26. Goldblatt J, Creegan R, Edkins T, Landau LI, Ryan G, Walpole IR. Mutation analysis of Western Australian families affected by cystic fibrosis. Med J Aust 1995;162:12-15.

27. Gilfillan A, Axton R, Brock DJ. Mass screening for cystic fibrosis heterozygotes: two assay systems compared. Clin Chem 1994;40:197-199.

28. Miedzybrodzka ZH, Yin Z, Kelly KF, Haites NE. Evaluation of laboratory methods for cystic fibrosis carrier screening: reliability, sensitivity, specificity, and costs. JMed Genet 1994;31:545-550.

29. Ferrie RM, Schwarz MJ, Robertson NH, Vaudin S, Super M, Malone G, Little S. Development, multiplexing, and application of ARMS tests for common mutations in the CFTR gene. Am J Hum Genet 1992;51:251-262.

30. Houdayer C, Cazeneuve C, Cougoureux E, Magnier C, Tredano M, Aymard P, Goossens M, Feldmann D. Clinical evaluation of the CF(12)m cystic fibrosis DNA diagnostic kit. Clin Chem 1998;44:1346-1348.

31. Brinson EC, Adriano T, Bloch W, Brown CL, Conway CC, Chen J, Eggerding FA, Grossman PD, Iovannisci DA, Madonik AM, Sherman DG, Tam RW, Winn-Deen 
ES, Woo SL, Fung S. Introduction to PCR/OLA/SCS, a multiplex DNA test, and its application to cystic fibrosis. Genet Test 1997;1:61-68.

32. Zielenski J, Rozmahel R, Bozon D, Kerem B-S, Grzelczak Z, Riordan JR, Rommens J, Tsui L-C. Genomic DNA sequence of the cystic fibrosis transmembrane conductance regulator (CFTR) gene. Genomics 1999;10:214-228.

33. Chamberlain JS, Gibbs RA, Ranier JE, Nguyen PN, Caskey CT. Deletion screening of the Duchenne muscular dystrophy locus via multiplex DNA amplification. Nucleic Acids Res 1988;16:11141-11156.

34. Kiesewetter S, Macek M, Davis C, Curristin SM, Chu CS, Graham C, Shrimpton AE, Cahsman SM, Tsui LC, Mickle J, Amos J, Highsmith WE, Shuber A, Witt DR, Crystal RG, Cutting GR. A mutation in CFTR produces different phenotypes depending on chromosomal background. Nat Genet 1993;5:274-278.

35. Chillon M, Casals T, Mercier B, Bassas L, Lissens W, Silber S, Romey MC, RuizRomero J, Verlingue C, Claustres $\mathrm{M}$ et al. Mutations in the cystic fibrosis gene in patients with congenital absence of the vas deferens. N Engl J Med 1995;332:14751480 .

36. Heim RA, Sugarman EA, Allitto BA. Improved detection of cystic fibrosis mutations in the heterogeneous US population using an expanded, pan-ethnic mutation panel. Genet Med 2001;3:168-176.

37. Beaudet AL and the Ad Hoc Committee on Mutation Nomenclature. Update on nomenclature for human gene mutations. Hum Mutat 1996;8:197-202.

38. Antonarakis SE and the Nomenclature Working Group. Recommendations for a nomenclature system for human gene mutations. Hum Mutat 1998;11:1-3 (http:// archive.uwcm.ac.uk/uwcm/mg/docs//mut_nom.html)

39. Sepulveda W, Leung KY, Robertson ME, Kay E, Mayall ES, Fisk NM. Prevalence of cystic fibrosis mutations in pregnancies with fetal echogenic bowel. Obstet Gynecol 1996;87:103-106.

40. Dicke JM, Crane JP. Sonographically detected hyperechoic fetal bowel: significance and implications for pregnancy management. Obstet Gynecol 1992;80:778-782.

41. Irish MS, Ragi JM, Karamanoukian H, Borowitz DS, Schmidt D, Glick PL. Prenatal diagnosis of the fetus with cystic fibrosis and meconium ileus. Pediatr Surg Int 1997; 12:434-436.

42. Hodge SE, Lebo RV, Yesley AR, Cheney SM, Angle H, Milunsky J. Calculating posterior cystic fibrosis risk with echogenic bowel and one characterized cystic fi- brosis mutation: avoiding pitfalls in the risk calculations. Am J Med Genet 1999;82: 329-335.

43. Bosco AF, Lieberman ES, Norton ME. Echogenic bowel and one cystic fibrosis mutation in the fetus: predictive of cystic fibrosis or coincidence? [abstract] J Genet Couns 1997;6:471-472.

\section{APPENDIX}

\section{Electronic and database information}

- ACMG Standards and Guidelines for Clinical Genetics Laboratories (http://www.acmg.net)

- ACMG Recommendations for Standards for Interpretation of Sequence Variations (http://www.acmg.net)

- GeneClinics profile at www.geneclinics.org

- National Cystic Fibrosis Foundation at www.cff.org

- CF Mutation Database at http://www.genet.sickkids.on.ca

- New York State Department of Health (www.wadsworth. org/labcert)

- College of American Pathologists Checklist (CAP) (www.cap.org)

- NIH-DOE Task Force on Genetic Testing (http://www.genome. gov/page.cfm?pageID = 10001733)

- American College of Obstetricians and Gynecologists (www.acog.org)

- NIGMS Human Genetic Cell Repository (locus.umdnj. edu/nigms/)

Disclaimer: These standards and guidelines are designed primarily as an educational resource for clinical laboratory geneticists to help them provide quality clinical laboratory genetic services. Adherence to these standards and guidelines does not necessarily ensure a successful medical outcome. These standards and guidelines should not be considered inclusive of all proper procedures and tests or exclusive of other procedures and tests that are reasonably directed to obtaining the same results. In determining the propriety of any specific procedure or test, the clinical molecular geneticist should apply his or her own professional judgment to the specific clinical circumstances presented by the individual patient or specimen. It may be prudent, however, to document in the laboratory record the rationale for any significant deviation from these standards and guidelines. 